\title{
ADITIVOS ALIMENTARES EM DIETA PARA FRANGOS DE CORTE SOB ESTRESSE INFECCIOSO POR COCCÍDEOS
}

\author{
(Additives in the diet of broiler chickens under infectious stress by coccidian)
}

Ione lolanda dos Santos ${ }^{1}$, Alexandre de Mello Kessler ${ }^{2}$, Juliano Fraga Mendes ${ }^{2}$, Raquel Valim Labres $^{2}$

'Universidade Federal do Oeste do Pará, Instituto de Ciência e Tecnologia Das Águas; ${ }^{2}$ Universidade Federal do Rio Grande do Sul. Departamento de Zootecnia

Corresponding author: ione_iolanda@hotmail.com

RESUMO: Um experimento foi conduzido para avaliar o efeito de diferentes aditivos na integridade intestinal e no desempenho de frangos de corte sob condições de estresse infeccioso. Utilizou-se 240 frangos Cobb de um dia de idade e quatro dietas experimentais: dieta controle sem aditivo, probiótico, óleos essenciais e antibiótico. Avaliou-se o ganho de peso (GP), consumo de ração (CR), conversão alimentar (CA), escores de lesão intestinal, altura (AV) e densidade de vilos (DV), profundidade de cripta (PC) e a relação vilos:cripta $(\mathrm{V}: \mathrm{C})$ nos segmentos intestinais. Aos 28 dias foi encontrado nos animais alimentados com a dieta com óleos essenciais maior $(P<0,05)$ escore de lesão. A menor $P C$ e a maior $(P<0,05)$ relação $\mathrm{V}: \mathrm{C}$ foram encontradas em animais que receberam antibiótico aos 21 e 28 dias. As dietas com probiótico e antibiótico possibilitaram maior $(P<0,05)$ CR e GP no período de 21 dias. Porém, os animais que receberam a dieta com probiótico não apresentaram melhor $(P<0,05) \mathrm{CA}$. Com exceção da CA aos 21 dias, as dietas analisadas não melhoraram $(P<0,05)$ o desempenho e o escore de lesão dos animais em relação à dieta controle.

Palavras-chave: Antibiótico; cripta; óleos essenciais; probiótico; vilos.

ABSTRACT: An experiment was conduct to evaluate the effect of different additives around intestinal integrity and performance from broilers by infectious stress. We used 240 broilers and 4 experimental diets: control diet without additives, probiotic, essential oils and antibiotic. Weight gain (WG),feed intake (FI), feed conversion (FC), as well as height, width and density of villi, crypt depth (CD) and the ratio of villi height by crypt depth $(\mathrm{V}: \mathrm{C})$ of the intestinal segments were evaluated. The essential oils diet at 28 days found major $(P<0.05)$ lesion score. The lowest $C D$ and highest $V$ : $C$ ratio $(P<0.05)$ found in animals received antibiotics at 21 and 28 days. Diets with probiotic and antibiotic provided higher $(P<0.05) \mathrm{FI}$ and WG in the 21 day period. However, animals receiving the probiotic diet did not present better $(P<0.05)$ FC. With the exception of FC at 21 days, the diets analyzed did not improve $(P<0.05)$ the performance and the lesion score of the animals in relation to the control diet.

Keywords: antibiotic; crypt; essential oils; probiotic; villi. 


\section{INTRODUCTION}

A sobrevivência e o bom desempenho das aves dependem da forma como o organismo animal obtém energia e compostos químicos. Para a obtenção adequada é necessário que o trato digestório apresente características estruturais funcionais desde a ingestão dos alimentos até a sua absorção (Romer e Parsons, 1981).

A absorção está intimamente relacionada aos processos ocorridos na mucosa intestinal, cujo desenvolvimento é decorrente de eventos citológicos primários envolvendo as células totipotentes localizadas na cripta e ao longo dos vilos, e a perda por descamação envolvendo os vilos (Paixão e Castro, 2016). Distúrbios na microbiota normal ou nas células epiteliais intestinais, causados por algum tipo de estresse, patógenos e substâncias químicas podem alterar a permeabilidade desta barreira natural, facilitando a invasão de patógenos e outras substâncias nocivas, modificando - metabolismo, a capacidade de digestão e absorção de nutrientes e causando ainda inflamações crônicas na mucosa intestinal (Palm et al., 2015). Consequentemente, ocorre diminuição das vilosidades, aumento do turnover celular e diminuição da atividade digestiva e absortiva (Visek, 1978; Boleli et al., 2002).

Como os processos de absorção de nutrientes são totalmente dependentes dos mecanismos que ocorrem na mucosa intestinal, a manipulação de microrganismos probióticos, substâncias antibióticos e óleos essenciais dentro do trato gastrintestinal tem sido usada com o objetivo de melhorar o desempenho e, consequentemente, a eficiência energética do intestino (Pournazari et al., 2017).

Nos últimos anos, o emprego de microorganismos intestinais e substâncias antibióticas têm proporcionado bons resultados na nutrição das aves, principalmente em condições experimentais. Porém, respostas diferentes são encontradas em testes realizados a campo e em estações experimentais, segundo as distintas condições de criação. A manutenção do ambiente de conforto térmico e os níveis de contaminação das dietas, das instalações e dos equipamentos não são totalmente controlados dentro das unidades produtivas. Visando experimentar uma situação de desvio das condições ideais de manejo, propõe-se neste trabalho, um estresse infeccioso por Eimerias sp, a frangos de corte alimentados com dietas contendo substâncias probióticas e antibióticas. Serão avaliados o escore de lesão, a morfometria intestinal e o desempenho das aves aos 14, 21 e 28 dias de idade.

\section{MATERIAL E MÉTODOS}

Todos os procedimentos experimentais foram aprovados pelo Comitê de Ética em Uso de Animais da Universidade Federal do Rio Grande do Sul, sob o protocolo $n^{\circ} 24156$.

O experimento foi conduzido com frangos, machos Cobb, a partir do primeiro dia de vida, alojados em 20 gaiolas com 12 aves cada. A temperatura ambiental e a umidade relativa do ar foram controladas $e$ registradas diariamente. Os animais receberam durante todo o período experimental água clorada e ração (a base de milho e soja) ad libitum, sendo este período dividido em duas fases: inicial (1 a 21 dias), quando as aves receberam uma dieta contendo 3050 $\mathrm{kcal} \mathrm{EM} / \mathrm{kg}$ de ração, $21 \%$ de proteína bruta, $1,29 \%$ de lisina, $0,57 \%$ de metionina, $0,95 \%$ de $\mathrm{Ca}$ e $0,45 \%$ de fósforo disponível; e na fase de crescimento (22 a 28 dias), as aves receberam uma dieta contendo 3150 $\mathrm{kcal}$ de EM/kg, 20\% de proteína bruta, 
$1,20 \%$ de lisina, $0,53 \%$ de metionina, $0,90 \%$ de $\mathrm{Ca}$ e $0,42 \%$ de fósforo disponível. Os outros níveis nutricionais foram aqueles recomendados por Rostagno et al. (2017).

Os animais foram pesados semanalmente e avaliou-se o ganho de peso (GP), o consumo de ração (CR) e a conversão alimentar (CA).

$O$ delineamento experimental foi o inteiramente casualizado, constituído por dietas que receberam quatro tratamentos: 1 -controle negativo, sem aditivo melhorador de crescimento; 2 probiótico, contendo $3,5 \times 10^{10} \mathrm{UFC} / \mathrm{g}$ de Enterococus faecium (35 g/ton); 3 mistura de óleos essenciais, extraído a partir de plantas de tomilho e cravo-daíndia (100g/ton) e; 4 - controle positivo constituído pelo antibiótico Bacitracina Metileno Dissalicilato (BMD)11\% (200 g/ton).

Para o desafio com a inoculação de coccídios, as aves foram inoculados, individualmente, via intra-esofágica, aos 14 dias de idade, com solução contendo $10 \times 10^{3}$ oocistos de Eimeria acervulina e $5 \times 10^{3}$ oocistos de Eimeria maxima.

Aos 21 e 28 dias de idade, foi realizado o abate de cinco aves por tratamento para a avaliação de escores de lesão e os segmentos do intestino delgado para as análises de morfometria. O escore de lesão foi baseado no grau de severidade, variando de 0 a 4, segundo técnica descrita por Johnson e Reid (1970): 0 - ausência de lesões, 1 estrias ou petéquias na mucosa, 2 presença de estrias brancas ou petéquias mais numerosas, 3 identificação de extensa hemorragia, 4 Além de hemorragia, identifica-se a presença de necrose.

As amostras do duodeno, jejuno e íleo foram fixadas em formalina tamponada a 10\%, cortadas transversalmente, colocadas paralelamente em cassetes, processados em aparelho histotécnico e incluídos em parafina. Cortes histológicos de 3 a 5 micrômetros foram obtidos em micrótomo rotatório e corados com hematoxilina-eosina (Montanari, 2016).

Nos diferentes períodos $(14,21 \mathrm{e}$ 28 dias de idade) cinco lâminas por tratamento foram feitas para cada segmento do intestino delgado. De cada lâmina foram efetuadas seis medidas de altura de vilos (AV), comprimento de vilos (CV), largura de vilos (LV), densidade de vilos (DV) e profundidade de cripta (PC), utilizando-se uma objetiva de duas vezes de aumento e uma régua acoplada à lente. Os resultados das leituras foram multiplicados por um fator de correção, para obtenção do valor em micrômetro. A altura de vilos foi medida a partir da região basal dos vilos, coincidente com a porção superior das criptas, até seu ápice. As criptas foram medidas da sua base até a região de transição entre a cripta e os vilos.

O estudo morfométrico foi realizado através do sistema analisador de imagem (Image-Pro plus 6.0) acoplado a um microscópio binocular.

Os dados de escores de lesão, morfometria, relação vilos:cripta, e desempenho foram submetidos à análise de variância pelo programa estatístico SPSS (2017) e na presença de diferença significativa foi realizada a comparação entre as médias dos tratamentos pelo teste de Tukey $(P<0,05)$ ou Kruskal-Wallis $(P<0,05)$.

\section{RESULTADOS E DISCUSSÃO}

\section{Estresse infeccioso por Eimerias $s p$}

Aos 14 dias de idade não se constatou qualquer lesão nos segmentos intestinais analisados. Este resultado já era esperado, uma vez que a inoculação com eimerias ainda não havia ocorrido. No entanto, após o desafio ocorrido, aos 21 dias de idade foi encontrada diferença $(P<0,05)$ entre os tratamentos para a variável escore 
de lesão, causado por Eimeria máxima, nos animais que receberam a dieta com óleos essenciais (Tab. 1). Ao contrário do que foi encontrado por Bozkurt et al. (2014, 2016), o grupo com óleos essenciais apresentou maior número de lesões em relação aos grupos dos demais tratamentos, indicando uma capacidade de recuperação menor ao estresse infeccioso. Aos 28 dias, os escores foram menores que aos 21 dias, mostrando uma regressão na infecção.

Tabela 1 - Escore de lesão em frangos de corte sob estresse infeccioso por Eimeria maxima e Eimeria acervulina aos 21 e 28 dias de idade.

\begin{tabular}{lcccc}
\hline & \multicolumn{2}{c}{ Eimeria maxima } & \multicolumn{2}{c}{ Eimeria acervulina } \\
\cline { 2 - 5 } Tratamentos & 21 dias & 28 dias & 21 dias & 28 dias \\
\hline Controle & $1,2^{1}$ & $0,4^{\mathrm{ab}}$ & 0,8 & 0,4 \\
Probiótico $^{1}$ & 1,3 & $0,4^{\mathrm{ab}}$ & 0,8 & 0,4 \\
Óleo essencial $^{2}$ & 1,4 & $0,5^{\mathrm{b}}$ & 0,4 & 0,4 \\
Antibiótico $^{3}$ & 0,6 & $0,2^{\mathrm{a}}$ & 0,0 & 0,4 \\
Probabilidade & 0,149 & 0,036 & 0,672 & 1,0 \\
CV\% & 3,50 & 3,30 & 2,8 & 2,5 \\
\hline
\end{tabular}

Médias na mesma coluna seguidas de letras distintas diferem significativamente pelo teste de Kruskal-Wallis (P<0,05). ${ }^{1}$ Enterococus $\left(3,5 \times 10^{10} \mathrm{UFC} / \mathrm{g}\right)$. ${ }^{2}$ Extraídos de Tomilho e cravo-da-índia - 100g/ton). ${ }^{3} \mathrm{BMD} 11 \%$. CV= coeficiente de variação.

\section{Morfometria intestinal}

A maioria dos processos digestivos e de absorção ocorre no intestino delgado e parte da digestão ocorre na superfície das vilosidades. A máxima capacidade da digestão e absorção acontece quando o animal apresenta uma grande área luminal, com altas vilosidades e enterócitos maduros (Macari e Maiorka, 2017). Sendo assim, parâmetros morfométricos como altura, largura e densidade de vilos e profundidade de criptas são importantes indicadores da saúde digestiva e da capacidade de absorção na mucosa intestinal.

No presente trabalho, não foram encontradas diferenças $(P<0,05)$ para altura, densidade e largura de vilos em nenhum segmento do intestino delgado das aves aos 14, 21 e 28 dias de idade. No entanto, foram verificadas diferenças $(P<0,05)$ na profundidade de cripta e na relação vilos:cripta no íleo aos 21 e 28 dias (Tab. 2).

A cripta é uma área onde as células totipotentes se dividem dando origem à reposição dos enterócitos nos vilos. A profundidade de cripta é um indicativo da intensa renovação celular existente nos vilos em resposta à perda de células pela descamação ou inflamações de patógenos e toxinas produzidas por eles. Quando a intensidade da renovação celular é aumentada, mais energia da dieta é requerida, ficando menos disponível para o crescimento do animal. A adição de antibiótico na dieta diminui a população microbiana e a quantidade de inflamações provocadas pela microbiota (Hill et al., 2017). Neste trabalho o grupo que recebeu antibiótico na dieta apresentou menor profundidade de crípta $(P<0,05)$ em relação aos demais grupos analisados. A relação vilos:crípta também se mostrou maior e traduziu-se em melhores respostas de desempenho (Tab. 3). Segundo Agyekum e Nyachoti (2017) o aumento na relação vilos:crípta é um indicador de melhor capacidade digestiva e absortiva do intestino delgado. Os resultados encontrados neste trabalho corroboram em parte 
com aqueles encontrados por Silva et al., (2009). Estes autores verificaram que os animais da dieta com antibiótico também apresentaram uma relação vilos:crípta maior $(\mathrm{P}<0,05 \%)$, quando comparados ao grupo da dieta com óleos essenciais, diferentemente dos resultados apresentados por Fernandes et al., (2017).

Tabela 2 - Parâmetros morfométricos do íleo de frangos de corte sob estresse infeccioso por Eimerias sp aos 21 e 28 dias de idade.

\begin{tabular}{lllllll}
\hline & \multicolumn{5}{c}{28 dias } \\
\cline { 2 - 7 } Tratamentos & AV $(\mu \mathrm{m})$ & PC $(\mu \mathrm{m})$ & V:C & AV $(\mu \mathrm{m})$ & PC $(\mu \mathrm{m})$ & V:C \\
\hline Controle & 730,20 & $175,25^{\mathrm{a}}$ & $4,17^{\mathrm{b}}$ & 1015,83 & $283,36^{\mathrm{a}}$ & $3,59^{\mathrm{b}}$ \\
Probiótico $^{1}$ & 733,97 & $177,94^{\mathrm{a}}$ & $4,12^{\mathrm{b}}$ & 1021,59 & $286,00^{\mathrm{a}}$ & $3,57^{\mathrm{b}}$ \\
Óleo essencial $^{2}$ & 728,73 & $175,50^{\mathrm{a}}$ & $4,16^{\mathrm{b}}$ & 1016,61 & $286,63^{\mathrm{a}}$ & $3,56^{\mathrm{b}}$ \\
Antibiótico $^{3}$ & 814,25 & $151,83^{\mathrm{b}}$ & $5,36^{\mathrm{a}}$ & 1025,65 & $247,12^{\mathrm{b}}$ & $4,15^{\mathrm{a}}$ \\
Probabilidade & 0,416 & 0,037 & 0,001 & 0,998 & 0,004 & 0,019 \\
CV\% & 12,38 & 10,10 & 15,51 & 8,09 & 8,33 & 10,48 \\
\hline
\end{tabular}

Médias na mesma coluna seguidas de letras distintas diferem significativamente pelo teste de Tukey $(P<0,05)$. ${ }^{1}$ Enterococus faecium ( $\left.3,5 \times 10^{10} \mathrm{UFC} / \mathrm{g}\right) .{ }^{2}$ Extraídos de Tomilho e cravo-da-índia (100g/ton). ${ }^{3} \mathrm{BMD} 11 \%$. CV= coeficiente de variação. $A V=$ =altura de vilos. $P C=$ profundidade de cripta. $V: C=$ relação vilo:cripta.

\section{Desempenho zootécnico}

Aos 14 dias de idade os grupos de animais não apresentaram diferenças $(P>0,05)$ nos parâmetros de desempenho entre nenhum tratamento analisado. No período de 14 a 21 dias (Tab. 3), as dietas com probiótico e antibiótico possibilitaram um maior $(P<0,05)$ consumo de ração e ganho de peso. No entanto, o consumo de ração em relação ao ganho de peso para o tratamento com probiótico não resultou em melhor conversão alimentar. O melhor desempenho dos animais obtido na dieta com antibiótico pode estar associado aos resultados de escore de lesão e morfometria (Tab. 1 e 2). Os animais que receberam antibiótico obtiveram menos incidência de lesões no epitélio intestinal e melhor resposta à sua recuperação, assim como menor profundidade de cripta e maior relação vilos:crípta, o que poderia contribuir para uma melhor eficiência digestiva. Comparando dietas semelhantes em frangos sob estresse infeccioso Santos et al., (2009), não encontraram diferença significativa de desempenho aos 21 dias. No entanto, pode-se encontrar na literatura eficiência de dietas, para frangos sob desafio, contendo óleos essenciais (Fernandes et al., 2017) e probióticos (Huff et al., 2015).

No período de 22 a 28 dias (Tab. 3) não foram observadas diferenças $(P<0,05)$ de ganho de peso e consumo de ração entre os tratamentos analisados. Porém, pode-se observar uma diferença significativa entre os tratamentos na conversão alimentar dos animais que receberam a dieta com antibiótico quando comparados com aqueles da dieta com óleos essenciais. Esses resultados diferem daqueles encontrados por Fernandes et al. (2017) e Huff et al. (2015), que não encontraram diferenças $(P<0,05)$ de desempenho entre as dietas com 0 antibiótico e os óleos essenciais.

Dietas com óleos essenciais costumam apresentar um efeito antibiótico, principalmente coccidiano (Silva et al., 2009; Fernandes et al., 2017). As piores respostas, nos parâmetros escore de lesão e 
morfometria, encontrados no presente trabalho podem ter determinado as baixas $(P<0,05)$ respostas de desempenho dos animais do grupo com óleos essenciais.

Os animais da dieta com probiótico mostraram uma pior conversão alimentar aos 21 dias de idade, mas melhoraram significativamente aos 28 dias quando comparados àqueles que receberam a dieta com antibiótico (Tab. 3). No entanto Abdelrahman et al. (2014) não observaram diferença na conversão alimentar durante os dois períodos analisados entre os animais que consumiram as dietas com probiótico e antibiótico. Porém, Huff et al. (2015) constatou que o uso de probiótico diminuiu a mortalidade e melhorou a conversão alimentar e o desempenho dos animais infectados por coccídios.

O desafio por coccídio executado neste trabalho foi capaz de produzir alterações intestinais (Tab. 1 e 2), contudo as respostas alcançadas no desempenho zootécnico deixam dúvidas se foram promovidas pelo efeito dos aditivos, uma vez que a dieta controle não se diferiu das demais nos parâmetros de desempenho (Tab. 3).

Tabela 3 - Desempenho zootécnico de frangos de corte obtidos no período de 14 a 21 e 22 a 28 dias de idade.

\begin{tabular}{lllllll}
\hline & \multicolumn{3}{c}{14 a 21 dias } & \multicolumn{3}{c}{22 a 28 dias } \\
\cline { 2 - 7 } \multicolumn{1}{c}{ Tratamentos } & GP $(\mathrm{g})$ & CR $(\mathrm{g})$ & CA & GP $(\mathrm{g})$ & CR $(\mathrm{g})$ & CA \\
\hline Controle & $505,64^{\mathrm{bc}}$ & $692,33^{\mathrm{b}}$ & $1,37^{\mathrm{ab}}$ & 523,11 & 897,87 & $1,72^{\mathrm{ab}}$ \\
Probiótico $^{1}$ & $523,58^{\mathrm{ab}}$ & $725,64^{\mathrm{a}}$ & $1,39^{\mathrm{b}}$ & 530,11 & 912,17 & $1,72^{\mathrm{ab}}$ \\
Óleo essencial $^{2}$ & $492,64^{\mathrm{c}}$ & $683,85^{\mathrm{b}}$ & $1,39^{\mathrm{b}}$ & 522,16 & 915,64 & $1,75^{\mathrm{b}}$ \\
Antibiótico $^{3}$ & $547,51^{\mathrm{a}}$ & $733,06^{\mathrm{a}}$ & $1,34^{\mathrm{a}}$ & 533,93 & 908,02 & $1,70^{\mathrm{a}}$ \\
Probabilidade & $<0,001$ & 0,002 & $<0,001$ & 0,414 & 0,377 & 0,014 \\
CV\% & 4,71 & 3,85 & 17,65 & 2,33 & 1,91 & 1,86 \\
\hline
\end{tabular}

Médias na mesma coluna seguidas de letras distintas diferem significativamente pelo teste de Tukey $(\mathrm{P}<0,05) .{ }^{1}$ Enterococus faecium $\left(3,5 \times 10^{10} \mathrm{UFC} / \mathrm{g}\right) .{ }^{2}$ Extraídos de Tomilho e cravo-daíndia $-100 \mathrm{~g} /$ ton). ${ }^{3} \mathrm{BMD} 11 \%$. GP=ganho de peso. $\mathrm{CR}=$ consumo de ração. $\mathrm{CA}=$ conversão alimentar. $\mathrm{CV}=$ coeficiente de variação.

\section{CONCLUSÃO}

Com base nos resultados encontrados neste estudo, conclui-se que as dietas com aditivos, não foram capazes de melhorar ou minorar os parâmetros de morfometria intestinal e de desempenho em relação a dieta controle.

\section{REFERENCES}

ABDELRAHMAN, W.; MOHNL, M.; TEICHMANN, K. et al. Comparative evaluation of probiotic and salinomycin effects on performance and coccidiosis control in broiler chickens. Poultry
Science. v.94, p.918-926, 2014. Disponível em: https://academic.oup.com/ps/articleabstract/93/12/3002/2730299. Acesso em: 10 de setembro de 2018.

AGYEKUM, A.K.; NYACHOTI, C.M. Nutritional and Metabolic Consequences of Feeding High-Fiber Diets to Swine: A Review. Animal Nutrition and Feed Science. v. 3, p.716-725, 2017.

BOLELI, I.C.; MAIORKA, A.; MACARI, $M$. Estrutura funcional do trato digestório. In: Macari, M.; Furlan, R.L.; Gonzales, E. editores. Fisiologia aviária aplicada a frangos de corte. Jaboticabal: Funep, 2002, p.75-96. 
BOZKURT, M.; EGE, G.; AYSUL, N.et al. Effect of anticoccidial monensin with oregano essential oil on broilers experimentally challenged with mixed Eimeria spp. Poultry Science. v.95, n.8, p.1858-1868, 2016. Disponível em: https://academic.oup.com/ps/articleabstract/95/8/1858/2588848. Acesso em: 10/09/2018.

BOZKURT, M.; AYSUL, N.; Kuçukyilmaz, K. Efficacy of in-feed preparations of an anticoccidial, multienzyme, prebiotic, probiotic, and herbal essential oil mixture in healthy and Eimeria spp.-infected broilers. Poultry Science. V.93, p.389-399, 2014. Disponível em: https://academic.oup.com/ps/articleabstract/93/2/389/1547094. Acesso em: 10/09/2018.

FERNANDES, J.I.M.; KOSMANN, R.C.; VIOTT, A.M. et al. Avaliação de extratos de plantas sobre a resposta imune, o desempenho produtivo e a morfometria intestinal de frangos de corte desafiados com Eimeria sp. Revista Brasileira de Saúde e Produção Animal. v.18, n.1, p.127-139, 2017. Disponível em: http://mc04.manuscriptcentral.com/rbspa -scielo http://dx.doi.org/10.1590/S151999402017000100012. Acesso em: 10/09/2018.

HILL, D.R.; HUANG, S.; NAGY, M.S. et al. Bacterial colonization stimulates a complex physiological response in the immature human intestinal epithelium. eLife. p.1-32, 2017.

HUFF, G.R.; HUFF, W.E.; RATH, N.C. et al. Efficacy of a novel prebiotic and a commercial probiotic in reducing mortality and production losses due to cold stress and Escherichia coli challenge of broiler chicks. Poultry Science. v.94, p.918-926, 2015. Disponível em: https://academic.oup.com/ps/articleabstract/94/5/918/1577958. Acesso em: 18 de setembro de 2018.
JOHNSON, J.; REID, W.M. Anticoccidial Drugs: Lesion scoring techniques in battery and floor-pen Experiments with chickens. Experimental Parasitology. v.28, p.30-36, 1970.

MACARI, M.; MAIORKA, A. Fisiologia das aves comercias. Jaboticabal : Funep, 2017. 806p.

MONTANARI, T. Histologia: texto, atlas e roteiro de aulas. 3. edição Porto Alegre : edição do autor, 2016. 229 p. Disponível em: http://www.ufrgs.br/livrodehisto/. Acesso em: 05/05/2017.

PAIXÃO, L.A.; CASTRO, F.F.S. A colonização da microbiota intestinal e sua influência na saúde do hospedeiro. Universitas: Ciências da Saúde. v.14, n.1, p.85-96, 2016.

PALM, N. W.; ZOETE, M. R.; FLAV ELL, R. A. Immune-microbiota interactions in health and disease. Clinical Immunology. v.59, p.122-127, 2015.

POURNAZARI, M.; QOTBI, A.A.A; SEIDAVI, A. et al. Prebiotics, probiotics and thyme (Thymus vulgaris) for broilers: performance, carcass traits and blood variables. Revista Colombiana de Ciências Pecuárias. v.30, p.3-10, 2017.

ROMER, A.S.; PARSONS, T.S. Anatomia Comparada. Ed. México : Iberoamericana, 1981. p.428.

ROSTAGNO, H.S.; ALBINO, L.F.T.; HANNAS, M.I. et al. Tabelas brasileiras para aves e suínos composição de alimentos e exigências nutricionais. $4^{a}$ edição Viçosa: UFV, 2017. p.488.

SANTOS, I.I.; KESSLER, A.M.; MENDES, J.F. et al. Aditivos melhoradores de crescimento sobre o desempenho de frangos de corte desafiados por coccídios. In: 21 CONGRESSO BRASILEIRO DE AVICULTURA E 27 CONFERÊNCIA 
FACTA, 2009, Porto Alegre. Anais do Prêmio Lamas 2009. Campinas: FACTA, 2009. CD-ROM. Nutrição de monogástrico.

SILVA, M.A.; PESSOTTI, B.M.S.; COLNAGO, G.L. et al. Intestinal mucosa structure of broiler chickens infected experimentally with Eimeria tenella and treated with essential oil of oregano. Ciência Rural. v.39, n.5, p.1471-1477, 2009. Disponível em: http://www.scielo.br/scielo.php?script=sc i arttext\&pid=S010384782009000500026. Acesso em: 10/09/2018.

STATISTICAL PACKAGE FOR THE SOCIAL SCIENCES (SPSS). Version 22.0.0.0. [Computer program]. Chicago: SPSS Inc.; 2017.

VISEK, W.J. The mode of growth promotion by antibiotics. Journal Animal Science. v.46, p.1447-1469, 1978. 